BULL. AUSTRAL. MATH. SOC.

VOL. 18 (1978), 455-460.

\title{
Note on asymptotic equivalence for measure differential equations
}

\section{Sree Hari Rao}

Two nonlinear ordinary differential systems, one of which contains impulses are considered in this note, and assuming the existence of bounded solutions, some results on asymptotic equivalence type correspondence between them are obtained. At the end an open question is posed.

In this note we consider the following differential systems

$$
\begin{aligned}
& x^{\prime}=F(t, x), \\
& D x=F(t, x)+G(t, x) D u,
\end{aligned}
$$

where F, G:J $J \times R^{n} \rightarrow R^{n}$. For notations we refer to [3]. Here $u: J \rightarrow R$ is a right continuous function of bounded variation on compact subsets of $J$ and the discontinuities $0=t_{0}<t_{1}<\ldots<t_{n}<\ldots$ of $u$ tend to $\infty$ as $n$ tends to $\infty$. In (2), $D x$ and $D u$ denote the distributional derivatives of $x$ and $u$ respectively and $D u$ can be identified with Lebesgue-Stieltjes measure and acts impulsively at the discontinuities of $u$. Equation (2), for this reason, is known as a differential system containing impulses, or a measure differential system.

Throughout we assume the existence of bounded solutions for the systems (1) and (2), for all $t \in J$. Here we propose to give a result which ensures that the systems (1) and (2) are asymptotically equivalent (for the definition see [1, p. 92]). We need the following conditions in our later discussion:

Received 31 March 1978. 
(C) for $(t, x, y) \in J \times R^{n} \times R^{n}$ and for $h>0$, there is an $\alpha>0$ such that $|x-y+h[F(t, x)-F(t, y)]| \leq(1-\alpha h)|x-y|$;

$\left(\mathrm{C}_{2}\right)$ for $(t, x) \in J \times R^{n},|G(t, x)| \leq W(t,|x|)$, where

$$
\begin{aligned}
& W: J \times J \rightarrow J, W(r, s) \leq W(r, t) \text { for } s \leq t \text {, and } \\
& \int_{0}^{\infty} W(s, c) d v_{u}(s)<\infty \text {, where } c>0 \text { is a constant. }
\end{aligned}
$$

The major advantage of this study is that, in addition to the system (1) being nonlinear, no differentiability conditions are placed on $F$. For stability and boundedness results we refer the reader to [3], [4], [5], and [6].

THEOREM. Assume that Conditions $\left(\mathrm{C}_{1}\right)$ and $\left(\mathrm{C}_{2}\right)$ hold. If $x$ and $y$ are bounded solutions of (2) and (1) on $J$ respectively, then

$$
\lim _{t \rightarrow \infty}|x(t)-y(t)|=0 \text {. }
$$

Proof. Let $M$ be a positive number such that $|y(t)| \leq M$ for all $t \in J$ and let $x(t)$ be a bounded solution of (2) with a bound $N$ such that $x(0)=y(0)$. Now $\left(\mathrm{C}_{2}\right)$ implies that $\int_{0}^{\infty} W(s, N) d v_{u}(s)$ is finite, and thus $\lim _{t \rightarrow \infty} \int_{t}^{\infty} W(s, N) d v_{u}(s)=0$

Let $L>0$ be such that $\int_{0}^{\infty} W(s, N) d v_{u}(s) \leq L$. Define a function $m(t)$ on $J$ by the relation

$$
m(t)=|x(t)-y(t)|
$$

Clearly $m(0)=0$. Since $u$ is differentiable on $\left[t_{k-1}, t_{k}\right)$, $k=1,2, \ldots, x$ is also differentiable on $\left[t_{k-1}, t_{k}\right), k=1,2, \ldots$

Let $h>0$ be sufficiently small and let $t \in\left[t_{k-1}, t_{k}\right]$. Now, by definition for $t \in\left[t_{k-1}, t_{k}\right]$,

$$
m^{\prime}(t)=\lim _{h \rightarrow 0^{+}} \frac{m(t+h)-m(t)}{h}
$$


and in view of $\left(C_{1}\right)$ and $\left(C_{2}\right)$, we have

$$
m^{\prime}(t) \leq-a m(t)+W(t, N)\left|u^{\prime}(t)\right| .
$$

Integrating ( 4 ) between $t_{k-1}$ and $t_{k}$,

(5) $m(t) \leq m\left(t_{k-1}\right) e^{-\alpha\left(t-t_{k-1}\right)}+e^{-\alpha t} \int_{\left[t_{k-1}, t\right]} e^{\alpha s} W(s, N)\left|u^{\prime}(s)\right| d s$.

At the discontinuous points $t_{k}, k=1,2, \ldots$, we have

$$
m\left(t_{k}\right) \leq\left|m\left(t_{k}\right)-m\left(t_{k}\right)\right|+\lim _{h \rightarrow 0^{+}} m\left(t_{k}-h\right) .
$$

Using Lemma 2.5 of [3] and (5), we obtain

(6) $m\left(t_{k}\right) \leq m\left(t_{k-1}\right) e^{-\alpha\left(t_{k}-t_{k-1}\right)}+w(t, N)\left|u\left(t_{k}\right)-u\left(t_{k}^{-}\right)\right|$

$$
+e^{-\alpha t_{k}} \int_{\left[t_{k-1}, t_{k}\right)} e^{\alpha s_{W(s, N)}\left|u^{\prime}(s)\right| d s .}
$$

A similar computation as in Theorem 3.1 of [3], together with the estimates (5) and (6) yields that, for $t \geq 0$,

$$
m(t) \leq m(0) e^{-\alpha t}+e^{-\alpha t} \int_{0}^{t} e^{\alpha s} W(s, N) d v_{u}(s)
$$

Since $m(0)=0$, we have

$$
\begin{aligned}
m(t)=|x(t)-y(t)| & \leq e^{-\alpha t} \int_{0}^{t} e^{\alpha s} W(s, N) d v_{u}(s) \\
& =e^{-\alpha t} \int_{0}^{t / 2} e^{\alpha s_{1}} W(s, N) d v_{u}(s)+e^{-\alpha t} \int_{t / 2}^{t} e^{\alpha s} W(s, N) d v_{u}(s) \\
& \leq e^{-\alpha t} e^{\alpha t / 2} \int_{0}^{t / 2} W(s, N) d v_{u}(s)+\int_{t / 2}^{t} W(s, N) d v_{u}(s) \\
& <e^{-\alpha t / 2} \int_{0}^{\infty} W(s, N) d v_{u}(s)+\int_{t / 2}^{\infty} W(s, N) d v_{u}(s) \\
& <L e^{-\alpha t / 2}+\int_{t / 2}^{\infty} W(s, N) d v_{u}(s) \\
& \rightarrow 0 \text { as } t \rightarrow \infty ;
\end{aligned}
$$


and the proof is complete.

COROLLARY. Let $\left(\mathrm{C}_{1}\right)$ and $\left(\mathrm{C}_{2}\right)$ hold. If $x_{1}$ and $x_{2}$ are bounded solutions of (2) on $J$ such that $x_{1}(0)=x_{2}(0)$, then (3) holds.

Proof. Let $y(t)$ be a bounded solution of (1) on $J$ such that $x_{1}(0)=y(0)=x_{2}(0)$. Then

$$
\left|x_{1}(t)-x_{2}(t)\right| \leq\left|x_{1}(t)-y(t)\right|+\left|y(t)-x_{2}(t)\right|,
$$

and the application of the previous theorem gives the desired result.

REMARK. Note that the corollary establishes the asymptotic uniqueness property of solutions of (2). More specifically, there may exist more than one bounded solution for (2) through the same point, but the difference between any two such solutions tends to zero as $t$ increases without bound.

EXAMPLE $(n=1)$. Consider the differential. equations

$$
y^{\prime}=-y+1, y(0)=2,
$$

and

$$
D x=-x+1+\left(1-x+e^{-2 t}\right) e^{-t} D u, x(0)=2,
$$

where

$$
u(t)=\left\{\begin{array}{lll}
0 & \text { for } & 0 \leq t<1, \\
t & \text { for } 1 \leq t<\infty .
\end{array} .\right.
$$

Note that $F$ and $G$ in $(7)$ and (8) satisfy the hypotheses $\left(c_{1}\right)$ and $\left(C_{2}\right)$. The solutions are given by

$$
y(t)=1+e^{-t}
$$

and

$$
x(t)= \begin{cases}1+e^{-t} & \text { for } 0 \leq t<1, \\ 1+e^{-t}+e^{-2 t} & \text { for } 1 \leq t<\infty .\end{cases}
$$

Clearly $x(t)$ and $y(t) \nrightarrow 0$ as $t \rightarrow \infty$; on the other hand 
$|x(t)-y(t)| \rightarrow 0$ as $t \rightarrow \infty$ and thus equations (7) and (8) are asymptotically equivalent.

The above discussion naturally poses the following question: What more conditions on $F$ and $G$ (in addition to $\left(C_{1}\right)$ and $\left(C_{2}\right)$ ) are required to prove the following statements:

(i) if $y$ is a bounded solution of (1) on $J$, then there exists a solution $x$ of (2) on $J$ such that (3) is satisfied, and

(ii) if $x$ is a bounded solution of (2) on $J$, then there is a solution $y$ of (1) on $J$ such that (3) holds.

In the case where (2) does not contain impulses, that is when (2) assumes the form $x^{\prime}=F(t, x)+G(t, x)$, (thus $u(t) \equiv t$ ) then a somewhat related answer is provided in [2]. The answer to the above question is still not known and certainly needs further investigation. If this is resolved then the results would improve and include many interesting results available in the literature (see [1], [2]).

\section{References}

[1] V. Lakshmikantham and S. Leela, Differential and integral inequalities. Volume I: Theory and applications (Mathematics in Science and Engineering, 55. Academic Press, New York and London, 1969).

[2] David Lowell Lovelady, "Asymptotic equivalence for two nonlinear systems", Math. Systems Theory 7 (1973), 170-175.

[3] M. Rama Mohana Rao and V. Sree Hari Rao, "Stability of impulsively perturbed systems", BulZ. Austral. Math. Soc. 16 (1977), 99-110.

[4] V. Sree Hari Rao, "Asymptotically self invariant sets and stability of measure differential equations", Nonlinear Anal. Theory, Methods App 2. 2 ( 1977), 1-7.

[5] V. Sree Hari Rao, "On boundedness of impulsively perturbed systems", Bulz. Austral. Math. Soc. 18 (1978), 237-242. 
[6] V. Sree Hari Rao, "Note on conditionally asymptotically invariant sets", J. Mathematical and Physical Sci. (to appear).

Department of Mathematics,

Osmania University,

Hyderabad,

Andhra Pradesh,

India. 